Proyecciones Journal of Mathematics

Vol. 28, No 2, pp. 141-153, August 2009.

Universidad Católica del Norte

Antofagasta - Chile

\title{
DISQUES J-HOLOMORPHES CONTENUS DANS UNE HYPERSURFACE
}

\author{
EMMANUEL MAZZILLI \\ UNIVERSITÉ DE LILLE 1 \\ Received : October 2008. Accepted : June 2009
}

\begin{abstract}
We study germs of J-Holomorphic curves contained in $M$, a real analytic hypersurface of an symplectic manifold of dimension 4. We show, under topological hypothesis on $M$, that if $M$ is compact then $M$ is of finite type and so there is no germs of J-holomorphic curves on $M$ (with $J$ adapted with the symplectic form). In $\mathbf{C}^{2}$ with the standard complex structure, this is a classical result of Diederich-Fornaess.
\end{abstract}

Subjclass [2000] : Primary 53D40, 53D12; Secondary 37D15. 


\section{Introduction et principaux résultats}

Considérons, $\left(\mathbf{R}^{4}, J\right)$ avec $J$ une structure presque complexe réelle analytique, $M=\{\rho=0\}$ un germe d'hypersurface réelle analytique en 0. Dans [1], nous avons développé une théorie du type régulier dans le cas non intégrable; pour énoncer précisément certains résultats, il faut rappeler quelques définitions et énoncés de [1] :

Definition 1.1. Soit $M=\{\rho=0\}$ un germe d'hypersurface lisse en 0; on dit que 0 est de type fini s'il existe $k \in \mathbf{N}$ tel que $: \forall \gamma$ disque $J$-holomorphe régulier $z \rightarrow \gamma(z), 0 \rightarrow 0$, l'ordre d'annulation de $\rho$ o $\gamma$ est inférieur à $k$. S'il $n$ 'existe pas de $k$ ayant la propriété ci-dessus, on dit que 0 est un point de type infini.

Definition 1.2. Si 0 est de type fini, on appelle type de $M$ en 0 , le plus petit des $k$ vérifiant la définition 1-1 et l'on note $\delta_{M}(0)$.

On établit dans [1], la proposition suivante:

Proposition 1.3. Si $M \subset \mathbf{R}^{4}$, alors $z \in M \rightarrow \delta_{M}(z)$ est une fonction semi-continue supérieurement.

Si 0 est de type infini, il semble raisonnable d'espérer trouver un germe de disque $J$-holomorphe, $\gamma$, contenu dans $M$; dans le cas d'une structure intégrable, ceci est vraie, voir par exemple [2], ou bien en remarquant qu'alors, la variété de Segre de $M$ en 0 a un ordre de contact infini avec $M$, (grâce à une application de l'inégalité de Lojasciewicz) et donc $\rho(0, z) \subset M$.

Quoiqu'il en soit, l'une et l'autre des méthodes utilisent fortement l'intégrabilité de la structure (par exemple, il n'y a pas l'equivalent des variétés de Segre dans le cadre preque complexe), et donc ne s'adaptent pas à notre situation. Pour contourner cette difficulté, nous sommes amenés à utiliser une version ad hoc (voir[4]et [5]) du théorème de Cartan-Kahler, en remarquant que 0 de type infini entraîne la "formelle intégrabilité" d'un certain système différentiel (que l'on imagine aisément, voir partie 3). On montre donc le théorème et le corollaire suivants :

Theorem 1.4. Soit $0 \in M \subset \mathbf{R}^{4}$, un germe d'hypersurface réelle analytique. Alors si 0 est de type infini, il existe un disque $\gamma$ contenu dans $M$, avec $\gamma(0)=0$.

Soit $E$ l'ensemble suivant:

$E:=\{X \in M / \exists \gamma$, disque holomorphe non trivial vérifiant $: \gamma(0)=X$ et $\gamma \subset M\}$. 
Corollaire 1.5. Sous les hypothèses du résultat précédent, E est fermé dans $M$.

Preuve du corollaire 1-5 :

Soit $\left(a_{n}\right)$ une suite de points de $E$ qui converge vers $0 \in M$ et $\left(\gamma_{n}\right)$ la suite des germes associés; si $\gamma_{n}$ est singulier en $a_{n}$, alors il existe une suite de points $\left(b_{k}\right)$ de $\gamma_{n}$ qui converge vers $a_{n}$, telle que $\gamma_{n}$ régulier en $b_{k}$. D'après la proposition 1-3, $a_{n}$ est donc un point de type régulier infini et par conséquent le théorème 1-4 entraîne l'existence de $\beta_{n} \subset M$ passant par $a_{n}$ et régulier. A nouveau, la proposition 1-3 assure que 0 est un point de type régulier infini, et le théorème 1-4 qu'il existe $\beta \subset M$ régulier passant par 0 .

En fait, il n'est point nécessaire de s'assurer que les germes associés aux points de $E$ sont réguliers; si $M$ est une hypersurface dans $\mathbf{R}^{4}$, elle ne peut contenir de germes de disques singuliers (mais pour obtenir ce résultat, il faut une étude plus fine des disques $J$-holomorphes contenus dans une hypersurface de $\mathbf{R}^{4}$; voir section 5 ).

Definition 1.6. On dit qu'une hypersurface $M$ dans une variété presque complexe $(V, J)$ est de type fini,s'il existe $k$ tel que tout point de $M$ est de type inférieur à $k$.

D'après la proposition 1-3, si $M$ est compacte et n'est pas de type fini, alors il existe $p_{0} \in M$ tel que $p_{0}$ est de type infini.

Nous avons également le résultat global suivant :

Theorem 1.7. Soit $(V, \omega)$ une variété symplectique réelle analytique de dimension 4 avec $\omega$ exacte sur $V$. Alors si $J$ est une structure presque complexe adaptée à $\omega$, toute hypersurface compacte $M$ est soit feuilletée, soit de type fini.

Corollaire 1.8. Sous les mêmes hypothèses que le théorème préceèdent et si de plus $M$ vérifie l'une des deux assertions suivantes : $\pi_{2}(M) \neq 0$ ou $\pi_{1}(M)$ est un groupe fini, alors toute hypersurface compacte $H$ est de type fini.

Preuve du corollaire 1-8 :

-Si $\pi_{1}(M)$ est un groupe fini, alors un théorème d'Haefliger assure qu'il n'existe pas sur $M$ un feuilletage réel analytique (voir [9], chapitre 6). 
-Si $\pi_{2}(M) \neq 0$, alors nous avons l'alternative suivante (car $M$ est de dimension 3, voir [9], chapitre 7, théorème de Novikov) pour un feuilletage $F$ de $M$ : soit il admet une composante de Reeb, ou bien toutes les feuilles sont compactes. Quoiqu'il en soit, il possède donc au moins une feuille compacte ; maintenant, intégrons la forme symplectique sur cette feuille $\omega$ et $J$ étant adaptées - d'un côté, cette intégrale est strictement positive et de l'autre elle est nulle à l'aide de la formule de Stokes ( $\omega$ est exacte sur $M)$.

Pour le cas de $\mathbf{C}^{2}$, il est inutile de faire des hypothèses topologiques sur $M$ pour éviter le cas feuilletée : ceci repose sur un argument de A.Glutsyuk (voir [3] et la section [5]). Nous avons donc le théorème suivant :

Theorem 1.9. Soit $\left(\mathbf{C}^{2}, \omega_{0}\right)$ avec $\omega_{0}$ la structure symplectique standard sur $\mathbf{C}^{2}$. Alors si $J$ est une structure presque complexe adaptée à $\omega_{0}$, toute hypersurface compacte est de type fini pour $J$.

Les théorèmes 1-7 et 1-9 reposent sur un résultat clef qui assure le prolongement des germes de disques $J$-holomorphes contenus dans une hypersurface de $\mathbf{R}^{4}$ définie au voisinage de 0 , en une courbe $J$-holomorphe fermée dans ce voisinage (voir théorème 5-2 de la section 5 ).

Pour $\omega$ une structure symplectique donnée, il existe toutjours des structures presques complexes adaptées. Par exemple, pour $\mathbf{C}^{2}$ muni de la structure symplectique standard alors la multiplication par $i$ est adaptée, et nous retrouvons donc, dans le cas de la dimension complexe 2, un résultat de Diederich-Fornaess ([8]).

Pour finir, citons un exemple d'application du théorème 1-7 : le cotangent d'une surface réelle analytique que l'on munit de sa structure symplectique naturelle, vérifie les hypothèses du théorème.

Mentionnons quelques questions ouvertes : pour le corollaire 1-5, on peut imaginer appliquer un théorème de compacité aux $\gamma_{n}$ mais au préalable il faut les prolonger, ce qui est possible d'après le théorème 5-2, mais qui ne nous permet pas de faire l'économie du théorème de Cartan-Kahler. De plus, il faut encore s'assurer que l'aire des disques prolongés est uniformément borné; dans le cas intégrable, ceci est possible en utilisant quelques résultats élémentaires sur la multiplicité des applications holomorphes (au moins dans le cas d'une hypersurface algébrique, voir [7]), ce qui n'a pas d'équivalent dans le cadre non intégrable.

Néanmoins, il nous semble intéressant de prouver s'il existe ou non un procédé effectif de reconstruction de $\gamma$ à la limite, à partir des $\gamma_{n}$, comme dans le cas intégrable (voir [7]). 
Enfin, il nous paraît naturel d'envisager ces problèmes en dimension plus grande - dans un premier temps, sous l'hypothèse $M$ pseudoconvexe -, la difficulté supplémentaire réside dans le fait suivant : on ne peut appliquer tel quel le résultat de Goldschmidt (théorème 4-2, section 4)car les projections ne sont plus surjectives, et donc le système n'est plus formellement intégrable (voir définition 4-1).

\section{Rappels et préliminaires}

Soit $D$ le disque unité de $\mathbf{R}^{2}, u: D \rightarrow \mathbf{R}^{4},(x, y) \rightarrow\left(u_{1}(x, y), \cdots, u_{4}(x, y)\right)$, $J$ une structure presque complexe sur $\mathbf{R}^{4}, J_{0}$ la structure complexe standard sur $\mathbf{R}^{2}$, on veut résoudre le système différentiel, $E$, au voisinage de zéro, suivant :

$$
\left\{\begin{array}{l}
d \rho_{u(x, y)} \operatorname{od} u_{(x, y)}=0 \\
d u_{(x, y)} J_{0}-J(u(x, y)) d u_{(x, y)}=0 .
\end{array}\right.
$$

Soit $J_{0}^{k}$ l'espace des k-jets en 0 ; on associe à $E$ le sous-espace $\varepsilon$ de $J_{0}^{1}$ défini par : $\theta \in \varepsilon$ si et seulement si $\theta$ vérifie $E$ en 0 . On considère également les prolongements de $E, E^{k} \forall k \geq 0$ (voir [4], P.137-140), obtenus par dérivation d'ordre $k$ de $E$ :

$$
\left\{\begin{array}{l}
D^{\sigma}\left(d \rho_{u(x, y)} o d u_{(x, y)}\right)=0 \\
D^{\sigma}\left(d u_{(x, y)} J_{0}-J(u(x, y)) d u_{(x, y)}\right)=0 .
\end{array}\right.
$$

où $\sigma=\left(\sigma_{1}, \sigma_{2}\right)$ avec $|\sigma| \leq k$, et on leur associe $\varepsilon^{k}$, le sous-espace de $J_{0}^{k+1}$ des jets qui vérifient $E^{k}$ en 0 .

Nous rappelons ici des résultats obtenus dans [1], dont nous aurons besoin par la suite pour la démonstration du théorème 1-4 :

Theorem 1.10. Considèrons $L^{p, q}:\left(\mathbf{R}^{2 n}\right)^{p+q+1} \rightarrow \mathbf{R}$, la forme de Levi d'ordre $p, q$ (voir [1], pour la définition précise).

Alors les deux propositions suivantes sont équivalentes:

(1) Il existe un disque $J$-holomorphe, $u$, régulier tangent à $M$ en 0 à l'ordre $k+2$.

(2) Il existe, $X$, champ de vecteur $J$-tangent à $M$ (ie une section régulière de $\left.T^{J}(M)\right)$ vérifiant :

$\forall(p, q),+q \leq k-1, L^{p, q}\left(X(0), X . X(0), \cdots, X^{p+q+1}(0)\right)=0$. 
De plus, on peut choisir $X$ tel que

$X(0)=\frac{\partial u}{\partial x}(0), \cdots, X^{k+1}(0)=\frac{\partial^{k+1} u}{\partial x^{k+1}}(0)$.

Definition 1.11. On définit la dérivée $(p, q)$-ième en 0 d'un champ de vecteurs $X$ sur $\mathbf{R}^{4}$,comme étant le vecteur $X$ dérivé $p$-fois dans sa propre direction et $q$-fois dans la direction $J X$

$$
D_{X}^{p, q} X(0)=(J X)^{q} X^{p} \cdot X(0)
$$

Definition 1.12. La collection de toutes les dérivées $(p, q)$-ième en 0,pour $0 \leq p+q \leq k$, d'un champ de vecteurs $X$ sur $\mathbf{R}^{4}$ est appelée son jet d'ordre $k$ et noté par $j_{0}^{k}(X)$. Ceci définie une application

$$
\left\{\begin{array}{l}
j_{0}^{k}: \Gamma\left(T \mathbf{R}^{4}\right) \rightarrow J_{0}^{k}\left(\mathbf{R}^{4}\right)=\left(\mathbf{R}^{4}\right)^{\frac{(k+1)(k+2)}{2}} \\
X \rightarrow\left(X(0), \ldots, D_{X}^{p, q} X(0), \ldots, D_{X}^{0, k} X(0)\right)
\end{array}\right.
$$

et le jet $\xi \in J_{0}^{k}\left(\mathbf{R}^{4}\right)$ est dit réalisable s'il appartient à $J_{0}^{k}(M):=j_{0}^{k}\left(\Gamma\left(T^{J} M\right)\right.$.

Proposition 1.13. Soit $\xi \in J_{0}^{k+1}\left(\mathbf{R}^{4}\right)$. On note $[\xi]_{k}$ sa $J_{0}^{k}\left(\mathbf{R}^{4}\right)$-composante et

$$
\left(\xi_{k+1,0}, \cdots, \xi_{p, q}, \cdots, \xi_{0, k+1}\right)
$$

sa partie homogène d'ordre $k+1$. Alors

$$
\exists X \in \Gamma\left(T^{J} M\right), j_{0}^{k+1}(X)=\xi
$$

si et seulement si

$$
\left\{\begin{array}{l}
\exists X_{1} \in \Gamma\left(T^{J} M\right), j_{0}^{k}\left(X_{1}\right)=[\xi]_{k} \\
\forall(p, q), p+q=k+1, \xi_{p, q}-\left[j_{0}^{k+1}\left(X_{1}\right)\right]_{p, q} \in T_{0}^{J} M .
\end{array}\right.
$$

Autrement dit, si $\xi$ est un $k+1$-jet réalisable, les composantes $N$ et $J N$ de sa partie homogène de degrés $k+1$, où $N$ est le vecteur normal à $M$ en 0 , sont entièrement déterminées par son jet d'ordre $k$; mais les composantes $J$-tangentes (toujours de la partie homogène d'ordre $k+1$ ) sont totalement libres.

Corollaire 1.14. Soit $u$ un disque $J$-holomorphe régulier au voisinage de 0 . Alors $u$ est tangent à $M$ en 0 à l'ordre $k+2\left(\right.$ soit $\left.\rho(u(z))=0\left(z^{k+2}\right)\right)$ si et seulement si son jet d'ordre $k+1$ est réalisable. 


\section{Prolongement du jet d'un disque tangent à $M$ à l'ordre $k+2$}

Proposition 2.1. Soient 0 un point de type infini et $\varepsilon^{k+1}$, pour tout $k \geq 0$, le prolongement d'ordre $k+1$ du système différentiel $E$. Alors la projection $\pi_{k}: \epsilon^{k+1} \rightarrow \epsilon^{k}$ est surjective pour tout $k \geq 0$.

Preuve de la proposition 2-1: Soit $u$ un disque $J$-holomorphe avec un ordre de contact $k+2$ avec $M$; montrer que $\pi_{k}$ est surjective revient à prouver l'existence de $v$, un disque $J$-holomorphe qui lui tangente à l'ordre $k+3$, avec $J_{0}^{k+1}(u)=J_{0}^{k+1}(v)$. On sait qu'il existe $\omega$, et donc $J_{0}^{k+2}(\omega)$, tel que $\omega$ à un ordre de contact $k+3$ avec $M$; d'après le théorème 1-10, on a par conséquent,

$$
L^{p, q}\left(\omega(0), \frac{\partial \omega}{\partial x}(0), \cdots, \frac{\partial^{p+q+1} \omega}{\partial x^{p+q+1}}(0)\right)=0,
$$

$\forall p+q \leq k$. Si $\phi$ est une fonction holomorphe au voisinage de 0 avec $\phi(0)=0$, alors $\omega o \phi$ a également un ordre de contact $k+3$ avec $M$, et donc pour tout $Y$ un champ de vecteurs $J$-tangent à $M$ de la forme $(\alpha+$ $\beta J) X_{\omega}$ (où $X_{\omega}$ est un champ de vecteurs associé à $\omega$ par le théorème 110 , et $\alpha, \beta$ sont des fonctions réelles régulières ), nous avons : $\forall(p, q)$ avec $p+q \leq k, L^{p, q}(Y)(0)=0$. Remarquons que $\operatorname{dim}_{\mathbf{C}}\left(T^{J}\right)=1$, et donc $X_{u}=(\tilde{\alpha}+\tilde{\beta} J) X_{\omega}$, ce qui entraîne, d'après ce qui précède, $\forall(p, q)$ avec $p+q \leq k, L^{p, q}\left(X_{u}\right)(0)=0$. A nouveau, le théorème 1-10 prouve l'existence d'un disque, $v, J$-holomorphe ayant un ordre de contact $k+3$ avec $M$ en 0 ; de plus, $J_{0}^{k+1}(v)=J_{0}^{k+1}(u)$ et $\frac{\partial^{k+2} v}{\partial x^{k+2}}(0)=X_{u}^{k+2}(0)$.

\section{Symbole d'un système différentiel}

Pour une description plus détaillé, on pourra consulter [4] et [5]. Afin d'éviter des notations un peu lourdes, nous nous contentons ici de rappeler uniquement ce dont nous aurons besoin. Considérons un système d'ordre 1 défini par :

$$
F_{l}\left(x, y, u_{i}(x, y), \partial_{1} u_{i}, \partial_{2} u_{i}\right)=0, \forall l \in\{1 \cdots, m\} .
$$

Pour $k \geq 1$, on définit le symbole de $\varepsilon^{k}, g^{k}$, qui à $\theta \in \varepsilon^{k}$ associe l'espace vectoriel

$g^{k}(\theta):=\left\{\left(\zeta_{k+1,0}, \cdots, \zeta_{p, q}, \cdots, \zeta_{0, k+1}\right) / \sum_{i} \zeta_{p+1, q-1}^{i} \partial_{1}^{i} F_{l}(\theta) \zeta_{p-1, q+1}^{i} \partial_{2}^{i} F_{l}(\theta)=0\right\}$. 
Ici, nous devons préciser que nous considérons $F$ comme une fonction des variables indépendantes $\left(x, y, u_{i}, p_{1}^{i}, p_{2}^{i}\right)$, et $\partial_{1}^{i} F_{l}, \partial_{2}^{i} F_{l}$ signifient respectivement $\frac{\partial F_{l}}{\partial p_{1}^{2}}, \frac{\partial F_{l}}{\partial p_{2}^{2}}$. Il est clair que $g^{k}(\theta)$ est entièrement déterminé par la partie homogène d'ordre 1 de $\theta$; pour cette raison, on considère $\theta \in \varepsilon \rightarrow g^{k}(\theta)$. Dans notre cas, nous savons (voir section précèdente) que les projections $\pi_{k}: \varepsilon^{k+1} \rightarrow \varepsilon^{k}$ sont surjectives, ce qui entraîne que $g^{k}(\theta)$ (pour $\theta \in \varepsilon$ ) peut être décrit de la manière suivante :

$$
\begin{aligned}
& g^{k}(\theta):=\left\{\left(\left(T_{1}-T_{2}\right)_{k+1,0}, \cdots,\left(T_{1}-T_{2}\right)_{p, q}, \cdots,\left(T_{1}-T_{2}\right)_{0, k+1}\right)\right\} \\
& \text { où }\left(T_{1}, T_{2}\right) \in \varepsilon^{k} \times \varepsilon^{k}, J_{0}^{k}\left(T_{1}\right)=J_{0}^{k}\left(T_{2}\right) \text { et } J_{0}^{1}\left(T_{1}\right)=J_{0}^{1}\left(T_{2}\right)=\theta
\end{aligned}
$$

et

$$
\left(\left(T_{1}-T_{2}\right)_{k+1,0}, \cdots,\left(T_{1}-T_{2}\right)_{p, q}, \cdots,\left(T_{1}-T_{2}\right)_{0, k+1}\right)
$$

désigne la partie homogène d'ordre $k+1$ de $T_{1}-T_{2}$. D'après le corollaire 1-14, ceci signifie que $T_{1}$ et $T_{2}$ sont des jets d'ordre $k+1$ réalisables ayant la même composante d'ordre $k$, nous pouvons appliquer alors la proposition 1-13 : la partie homogène de $T_{1}-T_{2} \in\left(T^{j} M\right)^{k+1}$. Nous venons donc de montrer $\left(\theta, g^{k}(\theta)\right)$ est un fibré vectoriel de fibre $\left(T^{J} M\right)^{k+1}$ au dessus de $\varepsilon$.

\section{Preuve du théorème 1-4}

Nous avons $\mathbf{R}^{4}$ muni d'une structure presque complexe $J$ dépendant analytiquement du point base et $M$ une hypersurface réelle analytique, par conséquent,le système $E$ de la partie 1 est à coefficients analytiques ; de plus d'après [5], $E$ vérifie:

Definition 4.1. On dit qu'un système différentiel $E$ est formellement intégrable si et seulement si $\left(\theta, g^{k+1}(\theta)\right)$ est un fibré vectoriel pour tout $k \geq 0$ au dessus de $\varepsilon$ et si de plus l'opérateur de restriction $\pi_{k}: \varepsilon^{k+1} \rightarrow \varepsilon^{k}$ est surjectif.

Theorem 4.2. Soit $E$ un système différentiel analytique formellement intégrable, alors pour tout $\theta \in \varepsilon^{k}$, il existe une solution analytique, $u$ de $E$, telle que $J_{0}^{k+1}(u)=\theta$.

Preuve du théorème 1-4 :

le point 0 est un point de type infini ce qui entraîne la formelle intégrabilité du système $E$, d'après les parties 2 et 3 ; le théorème $1-4$ est alors la conséquence directe du théorème 4-2. 
Remarque 4.3. Il est clair que même si l'hypersurface et la structure sont seulement de classe $C^{\infty}$, le système $E$ reste formellement intégrable.

\section{Preuve du théorème $\mathbf{1 - 7}$}

Dans cette partie, on convient de noter par une majuscule l'image géométrique d'un disque et par une minuscule le disque proprement dit.

Nous allons commencer par définir le prolongement d'un disque $J$ holomorphe:

Definition 5.1. Soit $u: D \rightarrow \mathbf{R}^{4}, 0 \rightarrow 0$ un germe de disque $J$-holomorphe dont l'image est incluse dans $\Omega$, un voisinage ouvert de 0 dans $\mathbf{R}^{4}$; on dit que $u$ admet un prolongement à $\Omega$ ou se prolonge à $\Omega$, s'il existe $w$ une courbe $J$-holomorphe fermée dans $\Omega$ dont l'image $W$ contient $U$, l'image de $u$.

Commencons par démontrer le théorème suivant:

Theorem 5.2. Soit $0 \in M$ une hypersurface réelle analytique de $\mathbf{R}^{4}$ définie sur $\Omega$; soit u un germe de disque $J$-holomorphe,régulier, passant par 0 et contenu dans $M$. Alors, si $M$ n'est pas feuilletée en courbe $J$-holomorphe, $u$ se prolonge à $\Omega$ en une courbe régulière $J$-holomorphe.

Preuve du théorème 5-2 : Soit $x_{0} \in \bar{U}$, on va montrer que $u$ se prolonge en une courbe $J$-holomorphe au voisinage de $x_{0}$. Considérons, $X$ un champ de vecteurs $J$-tangent à $M$ au voisinage de $x_{0}$ et $L_{\zeta}(X(\zeta), X(\zeta)):=L_{\zeta}$ la forme de levi en $\zeta \in M$ appliquée au vecteur $J$-tangent $X(\zeta)$. Clairement $U \subset\left\{L_{\zeta}(X(\zeta), X(\zeta))=0\right\} ; M$ n'étant pas feuilletée, $\left\{L_{\zeta}(X(\zeta), X(\zeta))=\right.$ 0 \} est de dimension 2 , et $U$ dans l'une des composantes irréductibles de cet ensemble réel analytique, disons $\{g(\zeta)=0\}$ ( $g$ étant l'un des facteurs irréductibles en $x_{0}$ de $L_{\zeta}$ ); d'après le corollaire $1-5$, il existe $\tilde{u}$, un germe de disque $J$-holomorphe en $x_{0}$, dont l'image $\tilde{U}$ est contenue dans $M$; nous allons montrer qu'en fait $\tilde{U} \subset\{g(\zeta)=0\}$. Nous avons vu dans la section 2 , que si $\theta$ est un germe de disque $J$-holomorphe en $Y$ qui a un ordre de contact $k+2$ avec $M$, alors on peut trouver un re-paramétrage, $\alpha_{k}^{\theta}$ tel que $\frac{\partial^{i}\left(\theta\left(\alpha_{k}^{\theta}\right)\right)}{\partial x^{i}}(0)=X^{i}(Y)$, pour tout $i \leq k$. Soit $k \in \mathbf{N}$ quelconque et $\left(x_{n}\right)$ une suite de points de $U$ qui tend vers $x_{0}$, on note $u_{x_{n}}$ le germe de disque en $x_{n}$ défini par $u$; choisissons $\alpha_{k}^{u_{x_{n}}}$ un re-paramétrage ayant la propriété ci-dessus; d'après ce qui précède, en passant à la limite sur les jets d'ordre $k$ des disques $u_{x_{n}}\left(\alpha_{k}^{u_{x_{n}}}\right)$, on obtient que le jet d'ordre $k$ compatible, dont 
les dérivées d'ordre inférieur à $k$ en $x$ sont données par $X^{i}\left(x_{0}\right)$, annule à l'ordre $k$ la fonction $g$. D'autre part, toujours en accord avec ce qui précède, je peux trouver un re-paramétrage $\alpha_{k}^{\tilde{u}}$ tel que $\frac{\partial^{i}\left(\tilde{u}\left(\alpha_{k}^{\tilde{u}}\right)\right)}{\partial x^{i}}(0)=X^{i}\left(x_{0}\right)$, pour tout $i \leq k$, ce qui entraîne $g\left(\tilde{u}\left(\alpha_{k}^{\theta}\right)\right)$ s'annule à l'ordre $k$ en $x_{0}$, et par conséquent $g(\tilde{u})$ également. Je peux effectuer cette opération pour tout $k$, ce qui implique $\tilde{U} \subset\{g=0\}$ et $u$ se prolonge en une courbe régulière au voisinage de $x_{0}$.

En prolongeant de proche en proche de la manière précèdente,on obtient le résultat en s'assurant néanmoins que si l'on revient en $x_{0}$, le germe de disque obtenu reste dans la même composante irréductible de $\left\{L_{\zeta}=0\right\}$, soit $\{g(\zeta)=0\}$; ceci est assuré car le jet du disque en question (quitte à re-paramétrer) est encore donné par le jet d'ordre $k$ compatible, dont les dérivées d'ordre inférieur à $k$ en $x$ sont les $X^{i}\left(x_{0}\right)$, qui annule $g$, d'après ce qui précède.

A présent, nous pouvons justifier l'affirmation suivante : (voir fin de la preuve du corollaire 1-5)

Remarque 5.3. $M$ ne peut contenir un germe de disque singulier en 0 .

Si $L_{\zeta}$ est identiquement nulle, alors $M$ est feuilletée en courbe régulière et l'affirmation est établie. Dans le cas contraire, $\left\{L_{\zeta}=0\right\}$ est de dimension

2 en 0 ; raisonnons par l'absurde, supposons qu'il existe $u$ singulier en 0 dont l'image $U \subset M$; alors $U \subset\{g=0\}$ où $\{g=0\}$ est la composante irréductible de $L_{\zeta}$ qui contient $U$. Les points singuliers d'un disque $J$ holomorphe sont isolés, ce qui entraîne qu'il existe une suite $\left(a_{n}\right)$ qui tend vers 0 avec $u$ qui est un germe de disque régulier en $a_{n}$; la preuve du théorème 5 -2 prouve alors que $\{g=0\}$ contient un germe de disque régulier en 0 et donc, est une variété réelle analytique de dimension 2 au voisinage de 0 , ce qui implique que $u$ est régulier en 0 . Nous avons également une version globale de cette affirmation :

Remarque 5.4. $M$ ne peut contenir une courbe $J$-holomorphe singulière (attention, $M$ pourrait contenir des courbes singulières sans contenir de germes de disques singuliers : il suffit que les composantes irréductibles de la courbe en un point multiple soient des disques réguliers).

En effet, supposons qu'il existe $C$ une courbe singulière dans $M$, alors $C$ contient un germe de disque régulier qui est lui-même contenu dans une courbe régulière, $C^{\prime}$, d'après le théorème 5 -2; mais alors $C$ et $C^{\prime}$ sont identiques, et donc $C$ n'a pas de singularités. 
Remarques :

Supposons que $M$ soit une hypersurface réelle analytique plongée(pas forcément compacte)dans une variété de dimension 4. Alors si $M$ est non feuilletée, la preuve du théorème 5-2 entraîne que tout germe de disque inclu dans $M$ se prolonge en une courbe fermée dans $M$. Maintenant si $M$ est feuilletée et simplement connexe, alors le germe de disque se prolonge par l'une des feuilles, mais toutes les feuilles d'un feuilletage transversalement réel analytique sont fermées([9], chapitre 6), et donc le germe se prolonge en une courbe fermée également. On a prouvé la proposition suivante :

Proposition 5.5. Soit $M$ une hypersurface d' une variété presque complexe $(V, J)$. Si $M$ est simplement connexe, alors tout germe de disque contenu dans $M$ se prolonge en une courbe fermée dans $M$

Fin de la preuve du théorème 1-7

Si $M$ n'est pas feuilletée, la preuve du théorème 5-2 assure que $U \subset C$ avec $c$ une courbe fermée régulière dans $M$, ce qui entraîne que $C$ est compacte sans bord. D'un côté - $J$ et $\omega$ étant adaptées - $\int_{C} \omega \geq 0$ et de l'autre $\int_{C} \omega=\int_{C} d \xi=0$ en utilisant la formule de Stokes; ce qui fournit une contradiction.

\section{Preuve du théorème 1-9}

Nous allons reproduire l'argument de A.Glutsyuk (voir aussi [3]). D'après le Théorème 1-7, il suffit de montrer que $M$ n'est pas feuilletée. Supposons que ce soit le cas :

le feuilletage ne dépend que de la structure $J$ sur une boule contenant $M$ car $M$ est compacte. Construisons $\tilde{J}$ une structure presque complexe sur $\mathbf{C} P^{2}$ adaptée à $\omega_{0}$ telle que: $\tilde{J}$ est la structure donnée sur cette boule et $\tilde{J}=J_{0}$, la structure complexe habituelle sur l'hyperplan à l'infini (la nouvelle structure n'est peut être pas réelle analytique mais on peut le faire dans la catégorie $C^{\infty}$, ce qui suffit pour ce qui suit).

Soit $\Omega$, l'ensemble des courbes rationnelles $\tilde{J}$-holomorphes rencontrant $T^{\tilde{J}} M$ et homologues à une droite projective $\mathbf{C} P^{1} \subset \mathbf{C} P^{2}$ (les courbes $\tilde{J}_{-}$ holomorphes homologues à $\mathbf{C} P^{1} \subset \mathbf{C} P^{2}$ sont appelées les $\tilde{J}$-droites); montrons que $\Omega$ est ouvert et fermé dans l'espace des $\tilde{J}$-droites.

1) $\Omega$ fermé: soit $D_{N}$ une suite de droites rencontrant $T^{\tilde{J}} M$ avec $D_{N}$ qui tend vers $D$, et $a_{N}$ une suite de points de $M$ tels que $D_{N}$ rencontre $T^{\tilde{J}} M$ 
en $a_{N}$; si $a$ une valeur d'adhérence de la suite $a_{N}$ ( $M$ est compacte), alors $a \in D$ et donc $D$ rencontre $T^{\tilde{J}} M$ en $a$.

2) $\Omega$ est ouvert : soit $D_{0}$ une droite de $\Omega$ et $a$ un point de $M$ tel que $D_{0}$ rencontre $T^{\tilde{J}} M$ en $a$. $F_{a}$, la feuille passant par $a$, est fermée dans une boule suffisamment petite autour de $a$. Si $D$ est une petite perturbation de $D_{0}$ alors l'indice d'intersection en $a$, de $F_{a}$ et $D$, est le même que celui de $D_{0}$ et $F_{a}$, donc strictement positif, ce qui entraîne : $D$ rencontre le feuilletage et $\Omega$ est ouvert.

D'après un résultat de Gromov ([6]), par deux points distincts de $\mathbf{C} P^{2}$, il passe une unique $\tilde{J}$-droite; de plus, si l'on note $D(a, b)$ la droite passant par $(a, b) \in \mathbf{C} P^{2} \times \mathbf{C} P^{2}$, l'application $(a, b) \rightarrow D(a, b)$ est régulière et donc l'ensemble des $\tilde{J}$-droites est connexe. Par conséquent, $\Omega$ est l' ensemble des $\tilde{J}$-droites, ce qui produit une contradiction car la $\tilde{J}$ droite à l'infini ne rencontre pas $T^{\tilde{J}} M, M$ étant compacte dans $\mathbf{C}^{2}$ et $\tilde{J}=J_{0}$ à l'infini.

\section{References}

[1] J. F. Barraud-E. Mazzilli. : Regular type of real hyper-surfaces in (almost) complex manifolds, Math-Zeit. 248, pp. 757-772, (2004).

[2] T. Bloom-I. Graham. : A geometric characterization of points of type $m$ on real submanifolds of $C^{n}$, J. Diff. Geometry. 12, pp. 171-182, (1977).

[3] B. Deroin. : Surfaces branchées et solénoides $\varepsilon$-holomorphes, arXiv. 593, (2004).

[4] Geometry I. : Encyclopaedia of Math.Sciences R. V. Gamkrelidze (Ed), 28 (1991).

[5] H. Goldschmidt. : Integrability criteria for systems of non-linear partial differential equations, J. Diff. Geometry. 1, pp. 269-307, (1967).

[6] M. Gromov. : Pseudoholomorphic curves in symplectic manifolds, Invent. Math. 82, pp. 307-347, (1985).

[7] E. Mazzilli. : Germes d'ensembles analytiques dans une hypersurface algèbrique, Ark. Mat. 44, pp. 327-333, (2006). 
[8] K. Diederich-J. E. Fornaess. : Pseudoconvex domains with real analytic boundary, Ann. of Maths. 107, pp. 371-384, (1978).

[9] Camacho-Lins neto. : Geometric theory of foliations, Birkhauser, Boston, MA (1985).

\section{Emmanuel Mazzilli}

E. M.: UFR de Mathématiques

Université de Lille 1

59655 Villeneuve d'Ascq

France

e-mail : mazzilli@math.univ-lille1.fr 